\title{
SHARP TRACE INEQUALITIES FOR FRACTIONAL LAPLACIANS
}

\author{
AMIT EINAV AND MICHAEL LOSS \\ (Communicated by Michael T. Lacey)
}

\begin{abstract}
The sharp trace inequality of José Escobar is extended to traces for the fractional Laplacian on $\mathbb{R}^{n}$, and a complete characterization of cases of equality is discussed. The proof proceeds via Fourier transform and uses Lieb's sharp form of the Hardy-Littlewood-Sobolev inequality.
\end{abstract}

\section{INTRODUCTION}

In a widely cited paper [6], José F. Escobar proved the trace inequality

$$
\left(\int_{\mathbb{R}^{n-1}}|(\tau f)(x)|^{\frac{2(n-1)}{n-2}}\right)^{\frac{n-2}{n-1}} \mathrm{~d} x \leq C_{n} \int_{\mathbb{H}^{n}}|\nabla f(x, t)|^{2} \mathrm{~d} x \mathrm{~d} t .
$$

Here $\mathbb{H}^{n}$ is the $n$-dimensional upper half-space $\left\{(x, t): x \in \mathbb{R}^{n}, t>0\right\}$ and $(\tau f)(x)$ denotes the trace of the function $f$ on the boundary of $\mathbb{H}^{n}$. The constant $C_{n}$ is given by

$$
C_{n}=\frac{1}{\sqrt{\pi}(n-2)}\left(\frac{\Gamma(n-1)}{\Gamma\left(\frac{n-1}{2}\right)}\right)^{\frac{1}{n-1}}
$$

and is the sharp constant in (1.1). Moreover, there is equality in (1.1) if and only if $f$ is a multiple of the function

$$
\frac{1}{\left((a+t)^{2}+|x+b|^{2}\right)^{\frac{n-2}{2}}}, a>0, b \in \mathbb{R}^{n} .
$$

Escobar observed that the problem is conformally invariant; i.e., the problem can be mapped to the $n$-dimensional sphere using stereographic projection. He then proceeds by showing first that an optimizer exists and then, using the method of Obata, to prove that $f^{\frac{4}{n-2}} s$ ( $s$ is the standard metric on Euclidean space) is an Einstein metric and hence flat. An entirely different proof of this inequality was found by Beckner [2. He deduced this inequality from the spectral form of Lieb's sharp Hardy-Littlewood-Sobolev (HLS) inequality on the sphere. Inequality (1.1) is then recovered via stereographic projection.

Another way of getting Lieb's sharp HLS inequality into the picture was shown in 44. It is based on the simple and well-known observation that among all functions $f(x, t)$ in the upper half-space that have a given trace $g$, the harmonic function

$$
h(x, t):=e^{-\sqrt{-\Delta} t} g(x)
$$

Received by the editors May 20, 2011.

2010 Mathematics Subject Classification. Primary 35A23.

The authors were supported in part by NSF grant DMS-0901304.

(C)2012 American Mathematical Society Reverts to public domain 28 years from publication 
minimizes the Dirichlet integral

$$
\int_{\mathbb{H}^{n}}|\nabla f(x, t)|^{2} \mathrm{~d} x \mathrm{~d} t
$$

By Gauss' theorem,

$\int_{\mathbb{H}^{n}}|\nabla h(x, t)|^{2} \mathrm{~d} x \mathrm{~d} t=\int_{\partial \mathbb{H}^{n}} \frac{\partial h}{\partial n}(x, 0) h(x, 0) \mathrm{d} x=\int_{\mathbb{R}^{n-1}} g(x) \sqrt{-\Delta} g(x) \mathrm{d} x=:(g, \sqrt{-\Delta} g)$.

Thus, the inequality (1.1) takes the form of a Sobolev inequality for fractional derivatives,

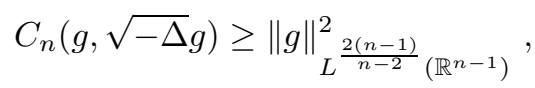

for which Lieb's sharp HLS inequality ([7]) yields the sharp constant, including all the cases of equality. Another important approach to Escobar's inequality is based on transportation theory [9]. This approach has been generalized in [10] replacing the two norms by general $L^{p}$-norms.

In view of these developments, it is natural to investigate the sharp trace inequalities for fractional Sobolev spaces. More specifically one may ask for the sharp constant for the inequality

$$
\left(\int_{\mathbb{R}^{n-1}}|(\tau f)(x)|^{\frac{2(n-1)}{n-2 \alpha}}\right)^{\frac{n-2 \alpha}{n-1}} \mathrm{~d} x \leq C_{n, \alpha}\left(f,(-\Delta)^{\alpha} f\right),
$$

where

$$
\left(f,(-\Delta)^{\alpha} f\right)=\int_{\mathbb{R}^{n}}|\widehat{f}(k)|^{2}(2 \pi|k|)^{2 \alpha} \mathrm{d} k
$$

As usual,

$$
\widehat{f}(k)=\int_{\mathbb{R}^{n}} f(x) e^{-2 \pi i x \cdot k} \mathrm{~d} x
$$

denotes the Fourier transform. There was an attempt in [11, where it was shown that for $\alpha \in(0,1)$ and $h$ defined by (1.4),

$$
\|g\|_{L^{\frac{2(n-1)}{n-1-2 \alpha}}\left(\mathbb{R}^{n-1}\right)}^{2} \leq C_{n, \alpha} \int_{\mathbb{H}^{n}}|\nabla h(x, t)|^{2} t^{1-2 \alpha} \mathrm{d} x \mathrm{~d} t
$$

where

$$
C_{n, \alpha}=\left(\frac{2^{1-4 \alpha}}{\pi^{\alpha} \Gamma(2-2 \alpha)}\right)\left(\frac{\Gamma((n-1) / 2-\alpha)}{\Gamma((n-1) / 2+\alpha)}\right)\left(\frac{\Gamma(n-1)}{\Gamma((n-1) / 2)}\right)^{\frac{2 \alpha}{n-1}}
$$

is the sharp constant. Using the Fourier transform in the variable $x$, it is easy to see that the right side of (1.11) can be written as

$$
2^{2 \alpha-1} \Gamma(2-2 \alpha)\left(g,(-\Delta)^{\alpha} g\right),
$$

and hence inequality (1.11) appears as a Sobolev inequality for the fractional Laplacian on $\mathbb{R}^{n-1}$ but not as a trace inequality for the fractional Laplacian on $\mathbb{R}^{n}$. Note that the harmonic function $h$ does not minimize the right side of (1.11). Minimizing the right side over all functions with a fixed boundary value $g$ yields a function $u(x, t)$ that satisfies the equation

$$
\Delta_{x} u+\partial_{t}^{2} u+\frac{1-2 \alpha}{t} \partial_{t} u=0, u(x, 0)=g(x) .
$$


The fractional Laplacian is then recovered by the formula

$$
\lim _{t \rightarrow 0} t^{1-2 \alpha} u(x, t)=-C(-\Delta)^{\alpha} g,
$$

where $C$ is some constant. This and general properties of functions that are 'harmonic' in this sense have been derived in [3].

The aim of our short paper is to extend the result of Escobar to true fractional Laplacians and prove a class of trace inequalities in their sharp form. This seems to be justified in view of the popularity of Escobar's result and of the simplicity of our proof and its generalizations.

It is customary to define the space $H_{\alpha}$ to be the space of all functions in $f \in$ $L^{2}\left(\mathbb{R}^{n}\right)$ whose Fourier transform $\widehat{f}$ satisfies

$$
\int_{\mathbb{R}^{n}}|\widehat{f}(k)|^{2}\left(1+|k|^{2}\right)^{\alpha} \mathrm{d} k<\infty
$$

The advantage of this definition is that $f$, being in $L^{2}\left(\mathbb{R}^{n}\right)$, is automatically a function. Here we are forced to take another route. For $2 \alpha<n$ we define the space $D_{\alpha}\left(\mathbb{R}^{n}\right)$ as the space of tempered distributions in $\mathcal{S}^{\prime}\left(\mathbb{R}^{n}\right)$ whose Fourier transform is a function in $L^{2}\left(\mathbb{R}^{n},|k|^{2 \alpha} \mathrm{d} k\right)$. More precisely, it consists of elements $T \in \mathcal{S}^{\prime}\left(\mathbb{R}^{n}\right)$ with the property that there exists a function $\widehat{f} \in L^{2}\left(\mathbb{R}^{n},|k|^{2 \alpha} \mathrm{d} k\right)$ such that for all $\phi \in \mathcal{S}\left(\mathbb{R}^{n}\right)$,

$$
\widehat{T}(\phi)=: T(\check{\phi})=\int_{\mathbb{R}^{n}} \widehat{f}(k) \phi(k) \mathrm{d} k .
$$

It is easy to see that $D_{\alpha}$ endowed with the norm

$$
\|f\|_{D_{\alpha}}:=\left(\int_{\mathbb{R}^{n}}|\widehat{f}(k)|^{2}|2 \pi k|^{2 \alpha} \mathrm{d} k\right)^{1 / 2}
$$

is a Hilbert space. Note, the factor $2 \pi$ is convenient since we can interpret the right side as

$$
\left(f,(-\Delta)^{\alpha} f\right)
$$

where $(f, g)$ is the standard $L^{2}\left(\mathbb{R}^{n}, \mathrm{~d} x\right)$ inner product. It is not difficult to see that $\mathcal{S}\left(\mathbb{R}^{n}\right)$ is dense in $D_{\alpha}\left(\mathbb{R}^{n}\right)$ and we will use this fact frequently. We shall see later that the tempered distributions in $D_{\alpha}$ are in fact functions in $L^{s}\left(\mathbb{R}^{n}\right)$ for a certain $s$, leading us to view $D_{\alpha}\left(\mathbb{R}^{n}\right)$ as a fractional homogeneous Sobolev space (see [1]).

For $f \in \mathcal{S}\left(\mathbb{R}^{n}\right)$ we define the restriction of $f$ to the $(n-m)$-dimensional hyperplane given by $\left\{x \in \mathbb{R}^{n}: x=\left(x_{1}, \ldots, x_{n-m}, 0,0, \ldots, 0\right)\right\}$ as

$$
f\left(x_{1}, \ldots, x_{n-m}, 0,0, \ldots, 0\right)=:\left(\tau_{m} f\right)\left(x_{1}, \ldots, x_{n-m}\right) .
$$

It is a standard result that $\tau_{1}$ extends to a bounded operator from $H_{\alpha}\left(\mathbb{R}^{n}\right)$ to $H_{\alpha-1 / 2}\left(\mathbb{R}^{n-1}\right)$. For the case where $f \in D_{\alpha}\left(\mathbb{R}^{n}\right)$ more can be said.

Theorem 1.1 (Sobolev trace inequality). Let $0 \leq m<n$ and $\frac{m}{2}<\alpha<\frac{n}{2}$. For any $f \in D_{\alpha}$ we have

$$
\left\|\tau_{m} f\right\|_{L^{\frac{2(n-m)}{n-2 \alpha}}}^{2} \leq C_{m, \alpha, n}\|f\|_{D_{\alpha}},
$$

where

$$
C_{m, \alpha, n}=2^{-2 \alpha} \pi^{-\alpha} \cdot \frac{\Gamma(n / 2-\alpha) \Gamma(\alpha-m / 2)}{\Gamma(\alpha) \Gamma(n / 2+\alpha-m)}\left\{\frac{\Gamma(n-m)}{\Gamma((n-m) / 2)}\right\}^{(2 \alpha-m) /(n-m)} .
$$


There is equality only if $f(x)$ is proportional to

$$
\int_{\mathbb{R}^{m}} \frac{1}{\left(\left|x^{\prime}\right|^{2}+\left|x^{\prime \prime}-y^{\prime \prime}\right|^{2}\right)^{(n-2 \alpha) / 2}} \frac{1}{\left(\gamma^{2}+\left|y^{\prime \prime}-a\right|^{2}\right)^{(n+2 \alpha-2 m) / 2}} d y^{\prime \prime}
$$

for some $a \in \mathbb{R}^{n-m}$ and $\gamma \neq 0$.

Note that for the case $\alpha=1$ and $m=1$ our constant differs from Esobar's by a factor $1 / 2$. This is due to the fact that our functions are defined on the whole space. For general values of $\alpha, D_{\alpha}\left(\mathbb{H}^{n}\right)$ is not defined unless one considers only functions that have support in $\mathbb{H}^{n}$. Also, in the case of $\alpha=1$ and $m=1$, (1.15) is easily computable and yields (up to a constant) the optimizer in Escobar's paper [6], as expected.

As mentioned above, trace theorems from $H_{\alpha}\left(\mathbb{R}^{n}\right)$ to $H_{\alpha-1 / 2}\left(\mathbb{R}^{n-1}\right)$ are standard. Theorem 2.4 in the next section yields the exact norm of the trace as a linear operator from $D_{\alpha}\left(\mathbb{R}^{n}\right)$ to $D_{\alpha-1 / 2}\left(\mathbb{R}^{n-1}\right)$. This, together with the sharp Sobolev inequality, will yield Theorem 1.1 which is, as far as we know, also new for integer $\alpha$, e.g., $\alpha=2$ and $n \geq 5$. The tools used in the various proofs, with the exception of Lieb's sharp Hardy-Littlewood-Sobolev inequality, are all elementary.

\section{Proof of Theorem 1.1}

That $f \in D_{\alpha}\left(\mathbb{R}^{n}\right)$ is in fact a function follows among other things from the next theorem.

Theorem 2.1 (Sobolev inequality). Let $0 \leq \alpha<n / 2$. For any $f \in D_{\alpha}\left(\mathbb{R}^{n}\right)$,

$$
\|f\|_{s}^{2} \leq 2^{-2 \alpha} \pi^{-\alpha} \frac{\Gamma(n / 2-\alpha)}{\Gamma(n / 2+\alpha)}\left\{\frac{\Gamma(n)}{\Gamma(n / 2)}\right\}^{2 \alpha / n}\|f\|_{D_{\alpha}}^{2},
$$

where $s=2 n /(n-2 \alpha)$. The constant on the right side of (2.1) is best possible and there is equality if and only if

$$
f(x)=A\left(\gamma^{2}+|x-a|^{2}\right)^{-(n-2 \alpha) / 2},
$$

where $A \in \mathbb{C}, \gamma \neq 0$ and $a \in \mathbb{R}^{n}$.

Remark 2.2. For $\alpha=1 / 2$, this theorem was proven in [8], Theorem 8.4, and is just the dual version of Lieb's sharp Hardy-Littlewood-Sobolev inequality. In a similar fashion, the theorem was proven in [5] for $\alpha<n / 2$ and for functions in $H_{\alpha}\left(\mathbb{R}^{n}\right)$. There is a slight subtlety in that for $\alpha \geq n / 4$ the Sobolev optimizers are not in $H_{\alpha}\left(\mathbb{R}^{n}\right)$. Thus, we repeat the simple proof for the case where the functions are in $D_{\alpha}\left(\mathbb{R}^{n}\right)$.

Remark 2.3. As a consequence of the above theorem and the density of $\mathcal{S}\left(\mathbb{R}^{n}\right)$ in $D_{\alpha}\left(\mathbb{R}^{n}\right)$ we have that any distribution in $D_{\alpha}\left(\mathbb{R}^{n}\right)$ is a function. Thus, we can say that $D_{\alpha}\left(\mathbb{R}^{n}\right)$ is the space of all functions $f \in L^{2 n /(n-2 \alpha)}\left(\mathbb{R}^{n}\right)$ for which there exists a function $g \in L^{2}\left(\mathbb{R}^{n},|k|^{2 \alpha} d k\right)$ such that

$$
\int_{\mathbb{R}^{n}} \phi(x) f(x) d x=\int_{\mathbb{R}^{n}} \widehat{\phi}(k) g(k) d k
$$

for all $\phi \in \mathcal{S}\left(\mathbb{R}^{n}\right)$. This function $g$ is unique and we shall denote it by $\widehat{f}(k)$. 
Proof. For $f, g \in \mathcal{S}\left(\mathbb{R}^{n}\right)$ we have that

$$
\int_{\mathbb{R}^{n}} \overline{f(x)} g(x) \mathrm{d} x=\int_{\mathbb{R}^{n}} \overline{\widehat{f}(k)} \widehat{g}(k) \mathrm{d} k,
$$

and by Schwarz's inequality,

$$
\int_{\mathbb{R}^{n}} \overline{\widehat{f}(k)} \widehat{g}(k) \mathrm{d} k \leq\|f\|_{D_{\alpha}}\left(\int_{\mathbb{R}^{n}} \frac{|\widehat{g}(k)|^{2}}{|2 \pi k|^{2 \alpha}} \mathrm{d} k\right)^{1 / 2} .
$$

Since

$$
\int_{\mathbb{R}^{n}} \frac{|\widehat{g}(k)|^{2}}{|k|^{2 \alpha}} \mathrm{d} k=\pi^{-n / 2+2 \alpha} \frac{\Gamma(n / 2-\alpha)}{\Gamma(\alpha)} \int_{\mathbb{R}^{n}} \int_{\mathbb{R}^{n}} \frac{\overline{g(x)} g(y)}{|x-y|^{n-2 \alpha}} \mathrm{d} x \mathrm{~d} y
$$

(see Corollary 5.10 in [8]), we may use Lieb's sharp form of the Hardy-LittlewoodSobolev inequality [7] (see also [8, 4]), which states that

$$
\int_{\mathbb{R}^{n}} \int_{\mathbb{R}^{n}} \frac{\overline{g(x)} g(y)}{|x-y|^{n-2 \alpha}} \mathrm{d} x \mathrm{~d} y \leq \pi^{n / 2-\alpha} \frac{\Gamma(\alpha)}{\Gamma(n / 2+\alpha)}\left\{\frac{\Gamma(n)}{\Gamma(n / 2)}\right\}^{2 \alpha / n}\|g\|_{r}^{2},
$$

where $r=2 n /(n+2 \alpha)<2$. There is equality if and only if

$$
g(x)=A\left(\gamma^{2}+|x-a|^{2}\right)^{-(n+2 \alpha) / 2},
$$

where $A \in \mathbb{C}, \gamma>0$ and $a \in \mathbb{R}^{n}$. Thus,

$$
\left(\int_{\mathbb{R}^{n}} \overline{f(x)} g(x) \mathrm{d} x\right)^{2} \leq 2^{-2 \alpha} \pi^{-\alpha} \frac{\Gamma(n / 2-\alpha)}{\Gamma(n / 2+\alpha)}\left\{\frac{\Gamma(n)}{\Gamma(n / 2)}\right\}^{2 \alpha / n}\|f\|_{D_{\alpha}}^{2}\|g\|_{r}^{2}
$$

and taking the supremum over all $g \in \mathcal{S}\left(\mathbb{R}^{n}\right)$ yields inequality (2.1) for $f \in \mathcal{S}\left(\mathbb{R}^{n}\right)$.

Now let $f \in D_{\alpha}\left(\mathbb{R}^{n}\right)$ be any distribution. Since $\mathcal{S}\left(R^{n}\right)$ is dense, there exists a sequence of functions $\widehat{f}_{j} \in \mathcal{S}\left(\mathbb{R}^{n}\right)$ with

$$
\int_{\mathbb{R}^{n}}\left|\widehat{f}_{j}(k)-\widehat{f}(k)\right|^{2}|k|^{2 \alpha} d k \rightarrow 0 .
$$

Thus, $f_{j}$ is a Cauchy sequence in $L^{s}\left(\mathbb{R}^{n}\right)$ with a limit which we call $\phi_{f}$. Since $\widehat{f}_{j}$ converges to $\widehat{f}$ in $\mathcal{S}^{\prime}\left(\mathbb{R}^{n}\right)$ and since the Fourier transform is an isomorphism on $\mathcal{S}^{\prime}\left(\mathbb{R}^{n}\right)$, we have that $f_{j}$ converges to $f$ in $\mathcal{S}^{\prime}\left(\mathbb{R}^{n}\right)$. Hence, $\phi_{f}=f$ and the inequality (2.1) is valid for all $f \in D_{\alpha}\left(\mathbb{R}^{n}\right)$. A similar approximation argument shows that equation (2.3) continues to hold for all functions $f \in D_{\alpha}\left(\mathbb{R}^{n}\right)$ and all $g \in L^{r}\left(\mathbb{R}^{n}\right)$. Hence, to establish the cases of equality we need equality in (2.4), which implies that

$$
\widehat{f}(k)=C \frac{\widehat{g}(k)}{|k|^{2 \alpha}},
$$

where $C$ is some constant and where $\widehat{g}$ is such that

$$
\int_{\mathbb{R}^{n}} \frac{|\widehat{g}(k)|^{2}}{|k|^{2 \alpha}} \mathrm{d} k<\infty .
$$

Further, there must be equality in the HLS inequality (2.6), which means that $g$ must be of the form (2.7). This function is integrable and smooth, and hence its Fourier transform is bounded with fast decay. Hence (2.9) also holds for this particular function. It remains to show that $f$ is of the form (2.2). To see this 
we imitate the proof of Corollary 5.9 in 8 and obtain that the inverse Fourier transform of $\widehat{g}(k) /|k|^{2 \alpha}$ is given by

$$
\pi^{-n / 2+2 \alpha} \frac{\Gamma(n / 2-\alpha)}{\Gamma(\alpha)} \int_{\mathbb{R}^{n}} \frac{g(y)}{|x-y|^{n-2 \alpha}} \mathrm{d} y .
$$

Since $g$ is an optimizer for the HLS inequality it must satisfy the Euler-Lagrange equation

$$
\int_{\mathbb{R}^{n}} \frac{g(y)}{|x-y|^{n-2 \alpha}} \mathrm{d} y=C g(x)^{r-1},
$$

where $C$ is some constant. This yields the form of the optimizer (2.2).

Theorem 2.4 (Trace inequality). Assume that $\frac{m}{2}<\alpha<\frac{n}{2}$. Then the trace $\tau_{m}$ has a unique extension to a bounded operator $\tau_{m}: D_{\alpha}\left(\mathbb{R}^{n}\right) \rightarrow D_{\alpha-m / 2}\left(\mathbb{R}^{n-m}\right)$. Moreover, for any $f \in D_{\alpha}\left(\mathbb{R}^{n}\right)$,

$$
\left\|\tau_{m} f\right\|_{D_{\alpha-m / 2}\left(\mathbb{R}^{n-m}\right)} \leq \frac{1}{2^{m} \pi^{\frac{m}{2}}} \frac{\Gamma\left(\frac{2 \alpha-m}{2}\right)}{\Gamma(\alpha)}\|f\|_{D_{\alpha}\left(\mathbb{R}^{n}\right)} .
$$

The constant in this inequality is best possible, and there is equality only if

$$
\widehat{f}\left(k_{1}, k_{2}\right)=C \frac{\widehat{g}\left(k_{1}\right)}{\left(\left|k_{1}\right|^{2}+\left|k_{2}\right|^{2}\right)^{\alpha}}
$$

with

$$
\int_{\mathbb{R}^{n-m}} \frac{\left|\widehat{g}\left(k_{1}\right)\right|^{2}}{\left|k_{1}\right|^{2 \alpha-m}} \mathrm{~d} k_{1}<\infty
$$

Here $k_{1} \in \mathbb{R}^{n-m}$ and $k_{2} \in \mathbb{R}^{m}$.

Proof. It is sufficient to prove the statement for $m=1$. The rest follows by iterating the result for $m=1$. Let $f \in \mathcal{S}\left(\mathbb{R}^{n}\right)$ and note that

$$
\widehat{\left(\tau_{1} f\right)}\left(k^{\prime}\right)=\int_{\mathbb{R}} \widehat{f}\left(k^{\prime}, k_{n}\right) \mathrm{d} k_{n} .
$$

By Schwarz's inequality,

$$
\begin{aligned}
& \left|\int_{\mathbb{R}} \widehat{f}\left(k^{\prime}, k_{n}\right) \mathrm{d} k_{n}\right|^{2} \\
= & \left|\int_{\mathbb{R}} \widehat{f}\left(k^{\prime}, k_{n}\right)\left(\left|2 \pi k^{\prime}\right|^{2}+\left(2 \pi k_{n}\right)^{2}\right)^{\alpha / 2}\left(\left|2 \pi k^{\prime}\right|^{2}+\left(2 \pi k_{n}\right)^{2}\right)^{-\alpha / 2} \mathrm{~d} k_{n}\right|^{2} \\
\leq & \frac{1}{2 \sqrt{\pi}} \frac{\Gamma\left(\alpha-\frac{1}{2}\right)}{\Gamma(\alpha)}\left|2 \pi k^{\prime}\right|^{-2 \alpha+1} \int_{\mathbb{R}}\left|\widehat{f}\left(k^{\prime}, k_{n}\right)\right|^{2}\left(\left|2 \pi k^{\prime}\right|^{2}+\left(2 \pi k_{n}\right)^{2}\right)^{\alpha} \mathrm{d} k_{n},
\end{aligned}
$$

which establishes (2.10) for all $f \in \mathcal{S}\left(\mathbb{R}^{n}\right)$ and hence the continuous extension of $\tau_{1}$ to all of $D_{\alpha}\left(\mathbb{R}^{n}\right)$.

Pick any $f \in D_{\alpha}\left(\mathbb{R}^{n}\right)$ and let $f_{j} \in S\left(\mathbb{R}^{n}\right)$ converge to $f$ in $D_{\alpha}\left(\mathbb{R}^{n}\right)$. Hence $\tau_{1} f_{j}$ converges to $\tau_{1} f$ in $D_{\alpha-1 / 2}\left(\mathbb{R}^{n}\right)$ and in particular in $L^{2(n-1) /(n-2 \alpha)}\left(\mathbb{R}^{n-1}\right)$ by Theorem 2.1. The formula

$$
\widehat{\left(\tau_{1} f_{j}\right)}\left(k^{\prime}\right)=\int_{\mathbb{R}} \widehat{f}_{j}\left(k^{\prime}, k_{n}\right) \mathrm{d} k_{n}
$$

holds for all $j$. Integrating this against a test function $\phi \in \mathcal{S}\left(\mathbb{R}^{n-1}\right)$ yields

$$
\int_{\mathbb{R}^{n-1}} \phi\left(x^{\prime}\right)\left(\tau_{1} f_{j}\right)\left(x^{\prime}\right) d x^{\prime}=\int_{\mathbb{R}^{n}} \widehat{\phi}\left(k^{\prime}\right) \widehat{f}_{j}\left(k^{\prime}, k_{n}\right) d k^{\prime} d k_{n} .
$$


Hence,

$$
\int_{\mathbb{R}^{n-1}} \phi\left(x^{\prime}\right)\left(\tau_{1} f\right)\left(x^{\prime}\right) d x^{\prime}=\int_{\mathbb{R}^{n}} \widehat{\phi}\left(k^{\prime}\right) \widehat{f}\left(k^{\prime}, k_{n}\right) d k^{\prime} d k_{n}
$$

and

$$
\widehat{\left(\tau_{1} f\right)}\left(k^{\prime}\right)=\int_{\mathbb{R}} \widehat{f}\left(k^{\prime}, k_{n}\right) d k_{n} .
$$

Thus, the steps in (2.13) are valid for any $f \in D_{\alpha}\left(\mathbb{R}^{n}\right)$ and the cases of equality follow from the cases of equality in Schwarz's inequality.

Proof of Theorem 1.1. The inequality follows from using Theorem 2.4 with $f$ and Theorem 2.1 with $\tau_{m} f$. In order to have equality we must have that $f$ satisfies (2.11) with (2.12). Also, $\tau_{m} f$ must satisfy (2.8), where $g$ is of the form (2.7) with the appropriate dimension. Since

$$
\widehat{\left(\tau_{m} f\right)}\left(k_{1}\right)=\int_{\mathbb{R}^{m}} \widehat{f}\left(k_{1}, k_{2}\right) \mathrm{d} k_{2}
$$

we conclude that $\widehat{f}$ is of the form (2.11) with $g$ of the form (2.7) with the appropriate dimension in the variables. This leads to (1.15).

\section{ACKNOWLEDGEMENTS}

We would like to thank Rupert Frank for helpful discussions concerning this work and Bruno Nazaret for pointing out reference [5].

\section{REFERENCES}

[1] Adams, David R. and Hedberg, Lars Inge: Function spaces and potential theory. Grundlehren der Mathematischen Wissenschaften [Fundamental Principles of Mathematical Sciences], 314. Springer-Verlag, Berlin, 1996. MR1411441 (97j:46024)

[2] Beckner, William: Sharp Sobolev inequalities on the sphere and the Moser-Trudinger inequality. Ann. of Math. (2) 138 (1993), no. 1, 213-242. MR1230930 (94m:58232)

[3] Caffarelli, Luis and Silvestre, Luis: An extension problem related to the fractional Laplacian, Comm. Partial Differential Equations 32 (2007), no. 7-9, 1245-1260. MR2354493 (2009k:35096)

[4] Carlen, Eric A. and Loss, Michael: Competing symmetries of some functionals arising in mathematical physics. Stochastic processes, physics and geometry (Ascona and Locarno, 1988), 277-288, World Sci. Publ., Teaneck, NJ, 1990. MR1124215 (92j:81338)

[5] Cotsiolis, Athanase and Tavoularis, Nikolaos K.: Best constants for Sobolev inequalities for higher order fractional derivatives. J. Math. Anal. Appl. 295 (2004), no. 1, 225-236. MR2064421 (2005d:46070)

[6] Escobar, José F.: Sharp constant in a Sobolev trace inequality. Indiana Univ. Math. J. 37 (1988), no. 3, 687-698. MR962929 (90a:46071)

[7] Lieb, Elliott H.: Sharp constants in the Hardy-Littlewood-Sobolev and related inequalities. Ann. of Math. (2) 118 (1983), no. 2, 349-374. MR717827(86i:42010)

[8] Lieb, Elliott H. and Loss, Michael: Analysis. Second edition. Graduate Studies in Mathematics, 14. American Mathematical Society, Providence, RI, 2001. MR 1817225 (2001i:00001)

[9] Maggi, F. and Villani, Cedric: Balls have the worst best Sobolev inequalities. II. Variants and extensions. Calc. Var. Partial Differential Equations 31 (2008), no. 1, 47-74. MR2342614 (2009f:46053) 
[10] Nazaret, Bruno: Best constant in Sobolev trace inequalities on the half-space. Nonlinear Anal. 65 (2006), no. 10, 1977-1985. MR2258478(2007m:46047)

[11] Xiao, Jie: A sharp Sobolev trace inequality for the fractional-order derivatives. Bull. Sci. Math. 130 (2006), no. 1, 87-96. MR2197182(2006i:46051)

School of Mathematics, Georgia Institute of Technology, Atlanta, Georgia 30332 0160

E-mail address: aeinav@math.gatech.edu

School of Mathematics, Georgia Institute of Technology, Atlanta, Georgia 303320160

E-mail address: loss@math.gatech.edu 